\title{
A lightweight wheelchair propulsion dynamometer for improving user energy efficiency and mobility
}

\author{
SJG Taylor, AT Symonds, J Gorjon, P Smitham, C Holloway \\ ASPIRE Centre for Rehabilitation Engineering and Assistive Technology \\ University College London \\ London U.K. \\ stephen.taylor@ucl.ac.uk
}

\begin{abstract}
The population of manual wheelchair users is growing; the vast majority of users are due to spinal cord injury, often presenting in young adults. Propulsion forces must be produced by the upper body only; such repetitive forces impact the shoulder and elbow joints, leading to long term pain and injury. This also has an effect on mobility, with consequential socio-economic implications. Training in the style of propulsion, especially from an early age, may lead to more efficient propulsion and reduced injury and pain. To measure the forces of propulsion and calculate energy expenditure, the connecting pins between a wheelchair pushrim and drivewheel were replaced with three multi-axis load cells able to measure the real time forces due to pushing. This paper reports the development of the Sensewheel Mk1 propulsion dynamometer: concept, load cell design and modelling, strain gauging and instrumentation, assembly, calibration and sample recorded data.
\end{abstract}

Keywords-wheelchair, forces, strain gauges, instrumentation, FE model, propulsion, load cell.

\section{INTRODUCTION}

It is estimated that $19 \%$ of the UK population live with a disability, of which approximately $10 \%$ use a manual wheelchair for mobility, which equates to approximately one million people [1]. People can become wheelchair dependent due to a sudden disability caused by Spinal Cord Injury (SCI), cerebrovascular accident or lower limb amputation, or due to progressive disability caused by osteoarthritis and neuromuscular diseases [2]. Under such circumstances, wheelchair provision can improve functional independence [3]; however insufficiency in terms of optimisation of the user and the wheelchair can lead to upper extremity dysfunction which can lead to reduced mobility and quality of life [2].

The wheelchair is a rehabilitation tool as well as a source of mobility for spinal cord injured people. Manual wheelchairs are generally preferred by younger people who have good upper body strength. There is limited evidence available to directly link the demands of manual wheelchair propulsion and shoulder joint pathology. What is known is that the prevalence of rotator cuff degeneration is greater in manual wheelchair users compared to aged matched controls [4] and that within a population of manual wheelchair users, rotator cuff degeneration is associated with increasing age and time as a wheelchair user [5]. In addition, models of tendon degeneration support the theory that tendon microtrauma is caused by repetitive overloading of the tendon [6]. It is therefore assumed that to minimise risk of injury, both repetition and peak force application should be minimised during manual wheelchair propulsion $[7,8]$. With this assumption in mind, there is a large body of biomechanical evidence available that has been used to both quantify upper limb demand during various propulsion tasks and also provide a measure of outcome to quantify the success of interventions. The biomechanical analysis methods used include measurement of force applied at the push rim using instrumented wheelchair wheels, measurement of joint kinematics using motion capture and inertial measurement systems, and also measurement of muscle activity levels and activation patterns using EMG. This experimental data has then been used to drive musculoskeletal models, to provide an estimate of both joint moments and joint contact forces.

Although at least two instrumented wheelchairs exist, there are none which are suitable for both laboratory and field use. The SmartWheel is capable of measuring three dimensional force and torque applied to the wheelchair push rim but weighs $4.08 \mathrm{~kg}$ [9]. The OptiPush instrumented wheelchair wheel also measures three dimensional force and torque, transmitting data via Bluetooth but weighs $5.7 \mathrm{~kg}$ [10]. The weight of the currently available instrumented wheelchair wheels is a major limitation, as the total weight addition to the chair, $8 \mathrm{~kg}$ to $11 \mathrm{~kg}$, has a significant impact on the forces required to push the chair [11]. To improve the validity of the biomechanical analysis of manual wheelchair propulsion, a lightweight instrumented wheelchair wheel would seem to be a necessity, a factor which led to the design reported here. One theme associated with this work is environmental accessibility of wheelchair users, another reason for requiring the instrumented wheels to be both lightweight and able to record data whilst on the move in the environment. A lightweight wheelchair pushrim wireless dynamometer (Sensewheel) was therefore designed and built to address these issues.

\section{METHODOLOGY}

\section{A. Specifications}

The requirements of the Sensewheel were that it should:

- $\quad$ Be able to measure static and dynamic 3D forces applied between the pushrim and drivewheel in real time;

- Measure, in the pushrim/drivewheel frame of reference, the 3 components of force applied by the hand to the 
pushrim: the (useful) tangential force, the radial force, and the force in the direction of the wheel axes (axial force);

- Incorporate a wireless data transmission system for data logging on a host PC, tablet or user's SmartPhone;

- Be lightweight enough that the user would not notice the difference between this and a standard chair;

- Be suitable for outdoor use as well as clinic; rain proof;

- Be eventually low-cost, thus enabling eventual widespread adoption into clinical use.

\section{B. Design concepts}

A wheel from a modern lightweight wheelchair (Van Os Excel 2) was adapted by replacing the usual 6 pillars connecting the pushrim to the drivewheel with three load cells (Fig. 1). A master / slave topology was used for each wheel whereby the three load cells (slaves) were connected to a master circuit using the $\mathrm{I} 2 \mathrm{C}$ protocol. Wires from each load cell were routed to the master unit mounted near the wheel hub containing a UHF radiotransmitter. The three components of the force vector developed between the pushrim and drivewheel were transmitted and measured by these load cells. The number of load cells (which carry all the load components between the pushrim and drivewheel) depends on the number of rim supports of the wheel being adapted. Sensewheels having three and four load cells were built initially, corresponding to the arrangement of interconnecting pillars supplied with the drive wheels, but later versions were restricted to wheels having 3 or 6 points of attachment, requiring only 3 load cells. The additional hardware required over an existing chair was minimised, since the relative weight of a load cell to the replaced pillar was almost negligible, and the only other additional component was the master circuit containing a lithium-ion cell, weighing 20g. The total additional weight of the Sensewheel over the standard wheel being adapted was $<100 \mathrm{~g}$. A UHF radio link (range $100 \mathrm{~m}$ ) was used to transmit the data from the wheel to a laptop PC hosting a LabView Graphical User Interface (GUI) for real time data visualisation and data logging.



Fig. 1. Sensewheel slave load cells mounted at 120deg intervals around the wheel, wired to the hub master, fitted to a VanOs Excel 2 wheelchair.

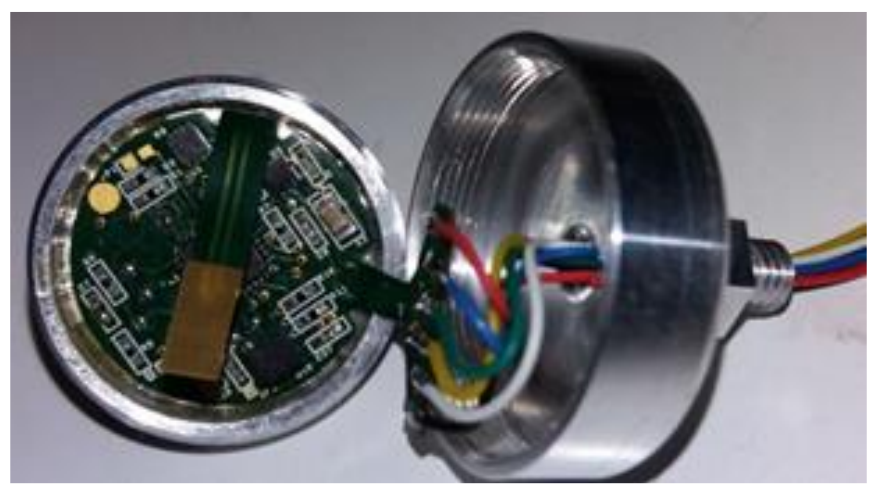

Fig. 2. Instrumented load cell constructed in two halves, to be screwed together and fixed with epoxy. Strain gauges lie underneath the flexi circuit.

\section{Load cell construction}

Each load cell was designed to measure the two orthogonal shear forces in the plane of the wheels, the axial force, and the axial torque. In order to minimise the height of the load cells, it would be important to choose sites for the gauges which would maximise the sensitivity to the measured forces and provide a clear distinction between the 3 forces being measured. Gauges were bonded to the inner diaphragm of one half which was screwed and bonded to the other half (Fig. 2). This arrangement would give good strain sensitivity and enable straightforward gauging. Four strain gauge half bridges (comprised of 8 off J5K-06-S105H-50C/DP foil gauges, Measurements Group UK) were arranged around the diaphragm, and exact gauge placement was determined by finite element analysis. When assembled, the Sensewheel would form a rigid construction such that (ideally) only forces and no torques would be developed at the load cell axes. However, the individual load cell torques were also measured to assess any deformation of the assembly during use.

As a diaphragm topology is unsuitable for separating shear forces from bending moments in the same plane, the measurements were restricted to the three forces and axial torque, all of which could be separated by suitable gauge placement and angles. A universal joint was used in line with the shaft of each load cell, recessed partially within the pushrim, to convert any applied bending out of the plane of the wheels into shear force, and the load cells were calibrated in shear and axial force only, using this same arrangement. For ease of assembly, and to minimise costs, the load cells were made in two halves which were screwed together after assembly. Aluminium alloy 7075 was used for the load cell bodies, for reasons of cost, low modulus and to keep the weight low. Strong epoxy was used to secure the halves and form an integral load cell.

\section{Finite element (FE) modelling}

The diaphragm behaviour under applied loads required careful gauge placement to avoid points of inflection, maximise sensitivity to each applied load, and provide sufficient strain separation between load directions 
(selectivity). A 3D axis-symmetric finite element model of the chosen mechanical design was carried out using COMSOL $^{\mathrm{TM}}$. Orthogonal direct and shear strains acting in the plane of the diaphragm, at several candidate radii, were output from the model post-process, in response to applied axial, shear and torque loads. These data were used to find the direct strains acting in any given direction and thus determine the optimum position and orientation of the gauges. Direct strains were rotated in software using standard plane strain formulae, in order to investigate and maximise the sensitivity and selectivity of each half bridge output in response to each applied load direction. Pairs of gauges simulating half bridges (of an inner and outer gauge) were tested using several candidate radii pairs, radially spaced at 90deg, Fig. 3. Curve fitting was used to assess the phase separation of half bridge outputs to each other for the orthogonal shear forces. In order to minimise the time taken to run multiple load cases, just two load cases were applied $(\mathrm{Fx}=100 \mathrm{~N}$ and $\mathrm{Fy}=100 \mathrm{~N})$ and combinations of these used to simulate 10deg increments of load resultant angle. Direct and shear strains from each of 8 sites (4 inner and 4 outer) were combined in the same proportions, to find the half bridge outputs at each angle increment. The radii of the inner and outer gauges were constrained by their size and bond pads, but the FE model highlighted the point of inflection to be avoided. Remaining choices were the angle of each gauge to the radius, and if these should be aligned or opposite to each other. These alternatives were simulated using the model.

\section{E. Instrumentation/circuit topology}

A distributed instrumentation system was designed in which each load cell contained a slave circuit (Fig. 2), connected to a master located near the hub of the wheel with 6 wires carrying power and signals. One Analog Devices ADuC7061 microcontroller ( $\mathrm{uC}$ ) was used in the master and in each slave. Each slave measured the voltages resulting from the 4 half bridges, at 50 samples/s per channel, and a 24 bit sigma-delta ADC was used to digitise these voltages with a noise-free resolution of 1 microstrain. The master $\mathrm{uC}$ polled the slaves for serial strain data via an I2C protocol, and then packed the data from each slave in a serial protocol for serial RS232 transmission to the remote computer via the UHF short

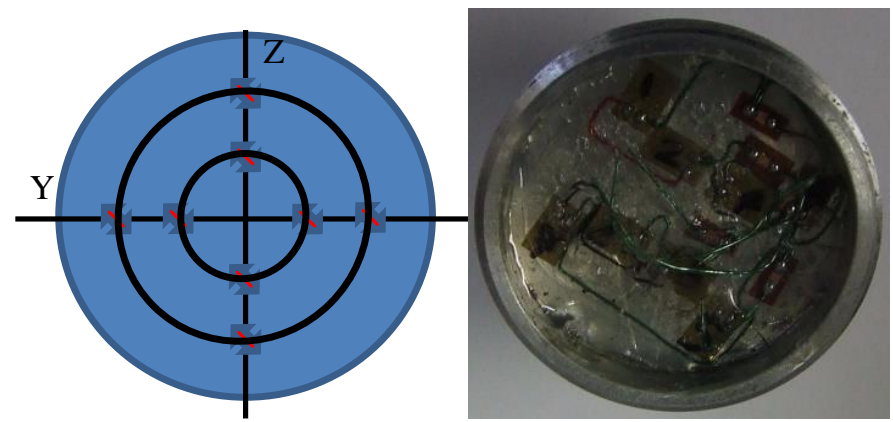

Fig. 3. Strain gauges were located on the end plate of the instrumented part of each slave, all aligned at $45 \mathrm{deg}$ to slave axes as shown (left) and wired as 4 half bridges (right); the gauge placement shown in the photograph was that originally used. range radio link. A LIS331DLH 3-axis digital accelerometer (Freescale) was included within the load cell and master circuits for orientation of the load cells and master in real time, in order to define the local axis system for referencing the shear forces, and to calculate the instantaneous wheel angle and push arc. Slave circuits were formed as a 4 layer polyimide flexible printed circuit (flexi), $20 \mathrm{~mm}$ in diameter, Fig. 2.

Strain gauges were wired to gauge bond pads (Fig. 2). A silicone adhesive was used to bond the flexi over the gauges and allow the gauge wires to protrude through holes in the polyimide for soldering to the flexi circuit. A programming port attached to a long wing of the flexi allowed the slave uC to be programmed with a slave identifier (1-3) prior to attachment in the load cell. Solder pads located on a further wing of the flexi allowed for wired connections to the master, exiting the load cell via a hole through the load cell axle. Once all electrical connections were made, the two parts of the load cell were screwed together, using an ' $\mathrm{O}$ ' ring to seal the internal cavity. Two-part epoxy adhesive was applied to the outer screw threads of the internal part prior to assembly, and excess removed prior to curing at $50 \mathrm{degC}$ overnight.

\section{F. Calibration}

Each load cell was individually calibrated (Fig. 4), at ambient temperature. A convenient method of calibrating for both axial and shear forces at once was devised. An arrangement was used whereby the load cell was held in a motorised chuck which was rotated at slow speed (typ. 1 rev per $90 \mathrm{~s})$. The motor/chuck/load cell was aligned at angle $\alpha(\sim$ 20deg) to the vertical such that with a load suspended from the axis of the load cell both an axial load component and a shear

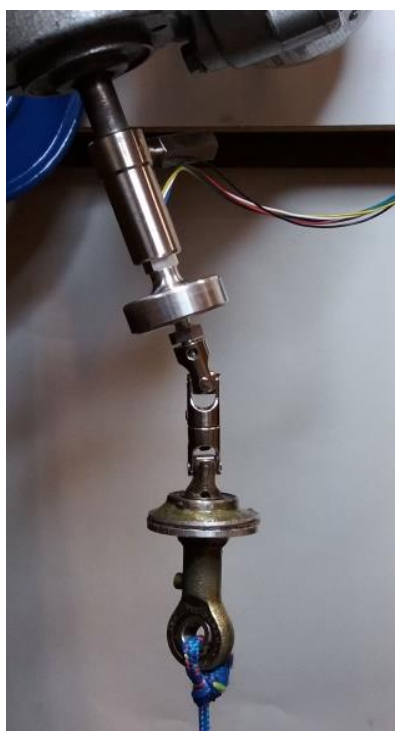

Fig. 4. Slave angled and rotated about axis to calibrate with known axial and shear forces.

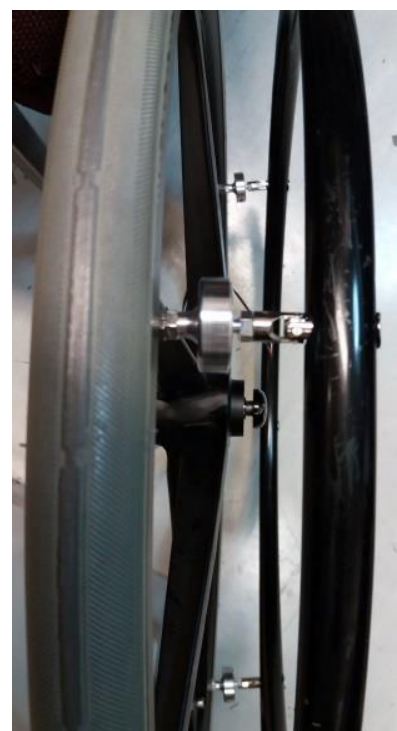

Fig. 5. Three slaves connecting pushrim with drivewheel. 
component were applied. In this way a sinusoidally-varying strain was experienced by each half bridge, allowing a best-fit sinusoid to be fitted to the signal for determining the sensitivities at any given axial angle. The accelerometer also included on the load cell flexi circuit allowed this sinusoidal profile to be referenced to the accelerometer axes (which were in turn used to determine the load cell angle at any given point in time in service). The slow speed of revolution minimised any applied torque due to inertial effects in the load application. The sinusoidal variation was due only to the shear force; the axial component was constant. A further record was made with no load applied, to obtain the zero load counts. The resulting output, obtained over one complete revolution of the motor, represented the sinusoidally-varying shear force at (90$\alpha$ ) deg to its normal direction on the load cell, and constant axial force at $\alpha$ deg to the load cell axis.

In order to separate the axial from the shear component, each strain channel was curve fitted to a sinusoid formed by the orthogonal components of the load cell accelerometer, and thus phase referenced to it. The mean value (subtracted from the zero load value) represented the axial component attenuated by $1 /(\cos \alpha)$, and the peak amplitude w.r.t. the mean value represented the maximum shear force sensitivity attenuated by $1 /(\sin \alpha)$. From these data, the axial and shear components and the shear phase shift w.r.t. the accelerometer 0 deg, were calculated.

Torque was applied in a separate calibration rig using a bending bar and dead weights. The sensitivity of each half bridge to each applied load (axial, shear at 0 deg, shear at 90 $\mathrm{deg}$, torque) was thus obtained, referenced to the accelerometer-defined axis system of the slave, and these values arranged in a $4 \times 4$ calibration matrix, which when inverted became the measurement matrix for that load cell.

When mounted onto Sensewheel (Fig. 5), each load cell's accelerometer was used to find its local angle at a known position of the wheel (load cell 1 placed at the top). These 3 angles were used to rotate the shear components about the axis of symmetry, $\mathrm{X}$, to find the shear forces along any given axis system, principally those tangential to, and radial to, the pushrim. The raw strain counts and accelerometer readings from each load cell were telemetered separately, then processed and combined in the GUI in real time.

\section{G. Data processing and Graphical User Interface (GUI)}

Strain and accelerometer data from each load cell and accelerometer data from the master were transmitted in real time as RS232 at 38000 Baud to the PC via UHF radio. A synchronisation protocol was used to decode the serial data. Upon reception they were first decoded using a $\mathrm{C}$ routine to separate the channels, and then presented as an array to a LabView GUI for processing. Data processing consisted of: converting incoming counts to slave $3 \mathrm{D}$ forces and torque in the axis system of the wheel, combining 3 slave forces to find the resultant tangential, radial and axial force on the pushrim.
The instantaneous push arc was also measured, displayed and recorded in the GUI. From these data the energy expenditure per push and the mean power over a session or journey are calculated.

The Sensewheel GUI enables real time propulsion data to be viewed simultaneously for right and left wheels. The GUI displays Fractional Effective Force (FEF, a measure of pushing efficiency), tangential force, linear velocity and push arc (Fig. 6). In advance of data capture, the wheel is positioned with load cell 1 on each wheel positioned at the top dead centre. A 'set zero' function is executed to record the angle of each load cell with respect to the coordinate system of the wheel. Data can then be recorded for a specified period and assigned an appropriate file name, and the raw data file is saved automatically.

The raw data file includes the accelerometer values for each load cell, the voltages from the half bridges presented in 'strain counts' and the raw data from the gyroscope in the plane of the wheel only. The raw data file is subsequently post-processed, to produce an output file yielding tangential, radial and axial forces, the moment about the wheel axle, FEF and push arc. Further parameters are readily computed such as wheel angle and power input to the pushrim.

\section{RESULTS}

Here we present the results obtained during development of the system, for both the FE and measured diaphragm strains, for applied axial and shear forces of $100 \mathrm{~N}$, and axial torque of $1 \mathrm{Nm}$, to illustrate agreement between the modelled and measured data. One data record is also presented for pushing using the calibrated wheel over an outdoor surface during a feedback study.

The following results are presented:

- 2D strains developed in the FE modelled diaphragm;

- $\quad$ modelled half bridge strain profiles, their amplitudes and

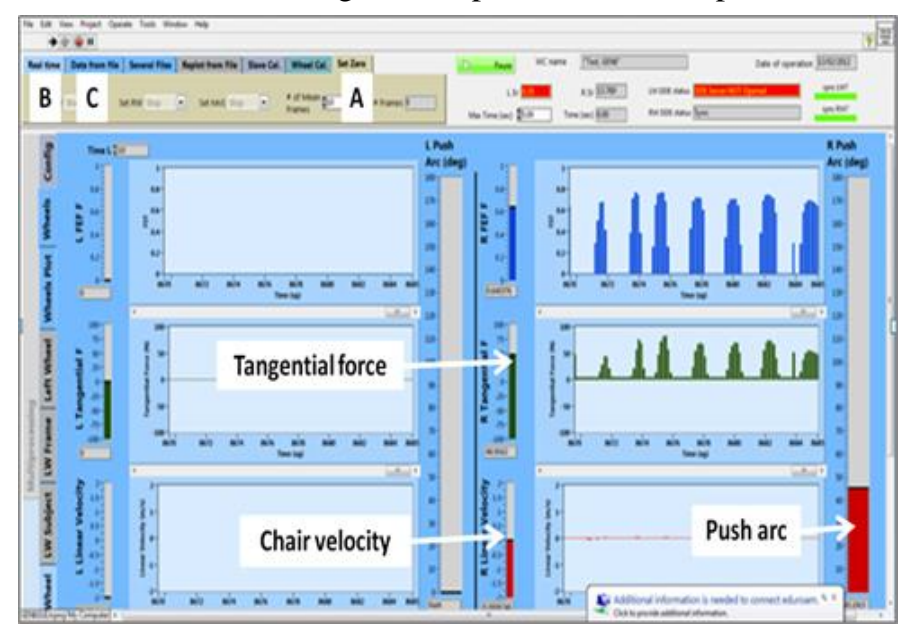

Fig. 6. LabView GUI front panel, with real time display of push efficiency (blue), useful tangential force (green), velocity, and push arc (red) From these are derived the push energy expenditure and mean power. 
phase relationships;

- $\quad$ measured half bridge strain profiles, their amplitudes and phase relationships;

- a 35s sample data log for outdoor propulsion showing tangential force and power: amplitude and phase.

\section{A. FE modelled strains on the diaphragm}

Fig. 7 shows the FE modelled plane strains along the $\mathrm{Y}$ (horizontal) and Z (vertical) axes, for $100 \mathrm{~N}$ applied axial force (along $\mathrm{X}$ axis, top pair) and $100 \mathrm{~N}$ applied shear force (along $\mathrm{Z}$ axis, bottom pair), looking down the $X$ axis of symmetry of the load cell. Axial force produced a uniform strain profile circumferentially, whereas shear force gave greater strains along the axis of shear, as expected. In both cases the diaphragm deformed to create external tensile and compressive stress near the periphery and hub respectively. Peak strains were \pm 3500 microstrain, occurring near the boss on the hub; this proved to be the most vulnerable point to breakage by excess shear force. These strain profiles (and numerate data) indicated a point of inflection at $6.5 \mathrm{~mm}$ radius in the direction of the applied shear force, and the gauges were therefore sited on $4.5 \mathrm{~mm}$ and $8.5 \mathrm{~mm}$ radii; in practice there was not much room for other positioning due to the space needed for bond pads.

\section{B. Modelled half bridge channel sensitivity and selectivity}

Half bridge outputs for 360deg shear loading, simulated by combining load directions as described, are shown in Fig.
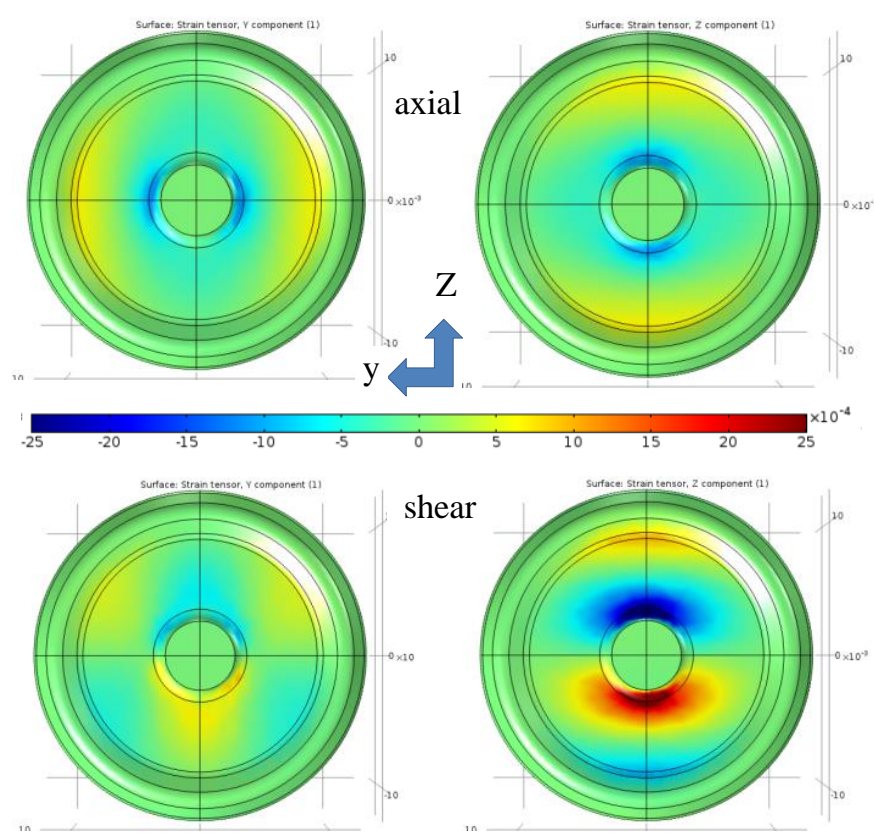

Fig. 7. Direct external strains developed in the $\mathrm{Y}$ direction (on left) and $\mathrm{Z}$ direction (on right) for $100 \mathrm{~N}$ force directed along $\mathrm{X}$ (axial) and $\mathrm{Z}$ (shear) axes (range $\pm 2.5 \mathrm{e}-3$ strain); internal strains are opposite in sign.
8. Gauge angles other than at $45 \mathrm{deg}$ to the radius gave profiles of different amplitude, therefore unequal sensitivity, and orienting the inner and outer gauges of each half bridge in the same direction gave best phase separation when at $45 \mathrm{deg}$ to the radius.

\section{Measured half bridge channel sensitivity and selectivity}

Digital counts corresponding to each half bridge output were logged for over one revolution of the motor, and referenced to the $\mathrm{X}$ axis of the accelerometer. Sinusoidal curve fitting was applied and Fig. 9 shows the resulting amplitudes and phase relationships of the 4 channels. The greater noise on these fitted waveforms is due to the unfiltered noise from the accelerometer axes, possibly due to vibration from the motor. The half bridge phase angles relative to ch1, from the FE model and from measurement, are shown in Table 1. The selected $45 \mathrm{deg}$ angle for the gauges gave both adequate sensitivity for foil strain gauges (resolutions of $\sim 6$ microstrain/ $\mathrm{N}$ for axial and $\sim 30$ microstrain/ $\mathrm{N}$ for shear)

\section{Sample data record}

Tangential rim force and power measured during a short outdoor trial (over flat concrete paving slabs) are shown in Fig. 10. Power (W) was measured as wheel moment $(\mathrm{Nm}) \mathrm{x}$ speed $(\mathrm{rad} / \mathrm{s})$. Negative excursions due to braking can be seen, especially at the end of the run.



Fig. 8. FE predicted half bridge strain profiles for 45deg gauges; strain vs 10deg intervals

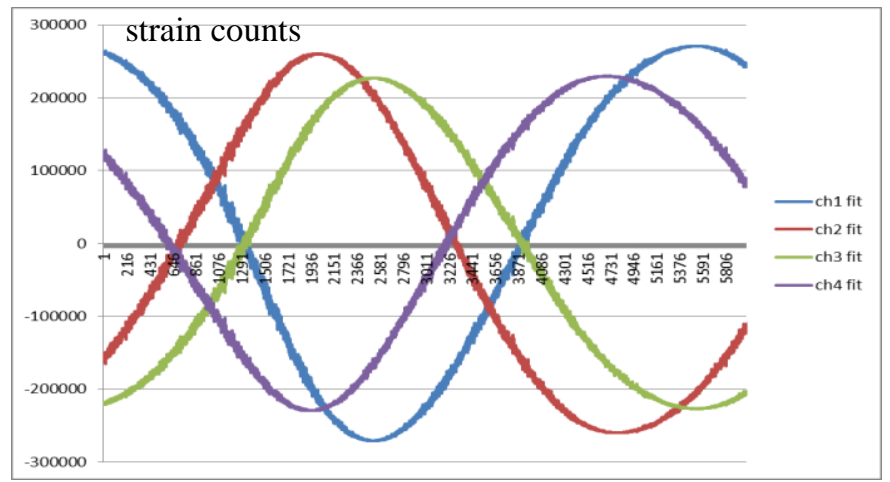

Fig. 9. Measured (and fitted) half bridge strain profiles for 45deg gauges; strain representative counts vs. $20 \mathrm{~ms}$ samples 
Table 1: The ideal, measured and FE modelled phase angles between half bridge channels (deg.)

\begin{tabular}{|c|c|c|c|}
\hline & Ideal angle & Measured angle & FE angle \\
\hline ch1 & 0 & 0 & 0 \\
\hline ch2 & -135 & -142 & -144 \\
\hline ch3 & 180 & 180 & 176 \\
\hline ch4 & 45 & 44 & 44 \\
\hline
\end{tabular}

\section{DISCUSSION}

A lightweight and unobtrusive laboratory tool for measuring the real time forces and power exerted whilst propelling a standard manual wheelchair has been described. Sensewheel is a self-contained lightweight dynamometer consisting of $3 \times 4$ d.o.f. load cells, able to be fitted to wheels having appropriate fixings, currently for laboratory use.

Three load cells are used to transmit the applied loads; a wheel-mounted distributed instrumentation system acquires, converts and transmits the data to a remote computer. A LabView GUI decodes and interprets these data as forces applied in tangential, radial and axial directions.

Strains from an FE model were extensively used and analysed to determine the optimum gauge locations in order to measure 4 degrees of freedom ( 3 forces and 1 torque) within a squat load cell body. The key objectives of adequate sensitivity and selectivity were met by testing gauge positions and angles. The measured relative force amplitudes and phases were found to be very similar to the FE predictions. It was found that a half bridge at each quadrant with gauge angles of $45 \mathrm{deg}$ to the radii, was effective in separating each applied load and providing adequate strain sensitivity to each load.

A novel method for calibrating the load cell strain axes against their inbuilt accelerometers was devised which allows for accurate calibration and repeatable setup. The load cell bodies were made of aluminium alloy, but the next version of Sensewheel will use titanium for greater robustness.

\section{CONCLUSIONS}

Wheelchair users, especially the young, need to be trained in efficient propulsion, to minimise the risk of upper limb injury in the long term, and to know what energy requirements are involved in traversing various terrains and slopes. A general purpose, lightweight, instrumented wheel has been developed to wirelessly record user 3D push forces, each push arc, speed and power in real time, to provide feedback for improving push style and efficiency. The Mk1 Sensewheel meets the main specification requirements, and was used in a $\mathrm{PhD}$ clinical study, aspects of which have been published elsewhere [12]. A Mk2 Sensewheel system is now under development, in which the load cells are wirelessly connected, for greater robustness and wider use.

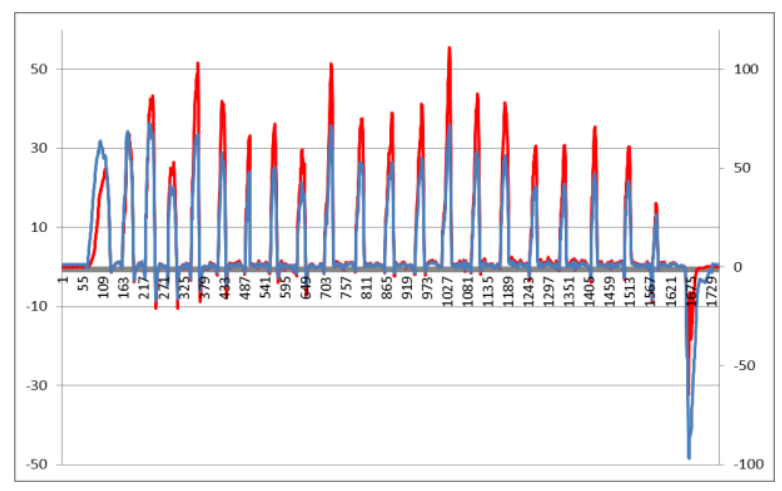

Fig. 10. Propulsion over level concrete; tangential force, $\mathrm{N}$ (blue, left axis) and power developed, W (red, right axis). Data samples at 50/s (x axis)

\section{REFERENCES}

[1] Papworth Trust 2016

http://www.papworthtrust.org.uk/campaigns/disability-facts-and-figures

[2] Requejo P. S., Furumasu J., Mulroy S. J. (2015). Evidence-based strategies for preserving mobility for elderly and aging manual wheelchair users. Top. Geriatr. Rehabil. 31, 26-41.10.1097/TGR.0000000000000042

[3] Hoenig, H., Landerman, L.R., Shipp, K.M., and George, L. (2003). Activity restrictions among wheelchair users. J Am Geriatr Soc, 51(9), 1244-1251.

[4] Akbar M, Balean G, Brunner M, et al. Prevalence of rotator cuff tear in paraplegic patients compared with controls. J Bone Joint Surg Am 2010; 92A(1): 23-30

[5] Akbar M, Brunner M, Balean G, et al. A cross-sectional study of demographic and morphologic features of rotator cuff disease in paraplegic patients. J Shoulder Elbow Surg 2011; 20(7): 1108-1113.

[6] Nho SJ, Yadav H, Shindle MK, et al. Rotator cuff degeneration - Etiology and pathogenesis. Am J Sports Med 2008; 36(5): 987-993.

[7] Boninger ML, Koontz AM, Sisto SA, et al. Pushrim biomechanics and injury prevention in spinal cord injury: Recommendations based on ULP-SCI investigations. J Rehabil Res Dev. 2005; 42: 9-19.

[8] Sawatzky B, DiGiovine C, Berner T, et al. The need for updated clinical practice guidelines for preservation of upper extremities in manual wheelchair users: a position paper. Am J Phys Med Rehabil. 2015; 94: 313-324.

[9] Cowan RE, Boninger ML, Sawatzky BJ, et al. Preliminary outcomes of the smart wheel users' group database: a proposed framework for clinicians to objectively evaluate manual wheelchair propulsion. Arch Phys Med Rehabil. 2008; 89: 260-268.

[10] Richter WM, Kwarciak AM, Guo L, et al. Effects of single variable biofeedback on wheelchair handrim biomechanics. Arch Phys Med Rehabil. 2011; 92: 572-577.

[11] Boninger ML, Cooper RA, Baldwin MA, et al. Wheelchair pushrim kinetics: body weight and median nerve function. Arch Phys Med Rehabil. 1999; 80: 910-915

[12] Holloway CS, Symonds A, Suzuki T, Gall A, Smitham P, Taylor SJG (2015). Linking wheelchair kinetics to glenohumeral joint demand during everyday accessibility activities. In: 2015 37th Annual International Conference of the IEEE Engineering in Medicine and Biology Society; 24782481. IEEE: Milan, Italy. 
